\title{
Percepción de la calidad de vida laboral de los trabajadores de los centros de salud familiar de la zona central de Chile
}

\author{
Miguel A. Bustamante ${ }^{1,2 *}$, Alejandra J. Álvarez ${ }^{3}$, Macarena E. Villalobos ${ }^{3}$ y Miguel I. Lucero ${ }^{3}$ \\ (1) Programa de Investigación de Excelencia Interdisciplinaria en Envejecimiento Saludable, PIEI-ES, \\ Universidad de Talca, Dos Norte 685, 3465548 Talca, Chile. (correo-e: mabu@utalca.cl) \\ (2) Facultad de Ciencias Económicas y Administrativas, de Universidad Católica Santiago de Guayaquil, \\ Av. Carlos Julio Arosemena Km. 11/2 vía Daule, Guayaquil, Ecuador. \\ (3) Facultad de Economía y Negocios, Universidad de Talca, Dos Norte 685, 3465548 Talca, Chile. \\ (correo-e: ale.alvarez.hor@gmail.com; mvillalobos13@alumnos.utalca.cl, mlucerob@alumnos.utalca.cl)
}

* Autor a quien debe ser enviada la correspondencia.

Recibido Jul. 19, 2019; Aceptado Sep. 11, 2019; Versión final Sep. 30, 2019, Publicado Jun. 2020

\begin{abstract}
Resumen
Se determina la percepción de la calidad de vida laboral (CVL) de los trabajadores de nueve establecimientos de atención primaria de salud en la Zona Central de Chile. La investigación se aborda bajo las perspectivas subjetiva y objetiva a través de un estudio cuantitativo, transversal, exploratorio, descriptivo y correlacional mediante la aplicación del cuestionario CVT-GOHISALO, que consta de 74 ítems aplicado a una muestra depurada de 303 trabajadores de la salud. Los resultados obtenidos muestran que cinco factores determinan la CVL, confirmado a través del análisis del modelo de medida de ecuaciones estructurales, que presenta un buen ajuste de los datos y muestra que existe un efecto directo entre los factores. Las relaciones de mayor incidencia fueron los constructos de seguridad y trabajo, apoyo y reconocimiento institucional respecto de desarrollo e integración. Los factores que inciden sobre la percepción de calidad de vida laboral son recreación y familia, apoyo y reconocimiento institucional, seguridad, bienestar laboral y desarrollo e integración.
\end{abstract}

\section{Perception of the quality of work life of the workers at family health centers of the central zone of Chile}

\begin{abstract}
The study aims to determine the quality of work life (QWL) perception of employees at nine primary health care facilities in the Central Zone of Chile. The research was performed under subjective and objective perspectives using a quantitative, cross-sectional, exploratory, descriptive, and correlational approach. The CVT-GOHISALO questionnaire (74 items) was applied to a sample of 303 health proffesionals. The results showed that five factors determine QWL. This was confirmed with the analysis of the measurement model of structural equations that showed good fitting of the data and a direct effect between the factors. The most influential relationships were the constructs of safety and work and the support and institutional recognition regarding development and integration. The factors that affected the perception of the quality of work life are recreation and family, support and institutional recognition, safety, work well-being, and development and integration.
\end{abstract}




\section{INTRODUCCIÓN}

El concepto de calidad de vida laboral, CVL, (Daubermann y Tonete, 2012), pone en valor un concepto de suma importancia a la hora de establecer relacionales laborales positivas (Segurado y Agulló, 2002), primero porque la actividad laboral es fundamental para el desarrollo de las personas (Granados, 2011), segundo, porque es parte constitutiva del ciclo de vida, CV (Pérez y Zurita, 2014), y tercero, porque posiciona el trabajo como un aspecto fundamental del desarrollo integral del individuo (Sirgy, et al., 2008). Es aquí donde el concepto de calidad de vida laboral toma fuerza considerando los factores incidentales que lo determinan y que se asocian, en gran medida, al bienestar subjetivo percibido por las personas y a los resultados que se observan a nivel de enfermería en la atención primaria de salud (Daubermann y Tonete, 2012). Todo ello se ve reflejado en la forma en que los trabajadores, principalmente los relacionados a la salud pública, perciben de su entorno laboral y que se puede expresar en estrés laboral que debe ser gestionada (Balducci, 2015) incrementando progresivamente la satisfacción laboral, SL, (Granados, 2011).

En consecuencia, conforme lo dicho, es menester gestionar la satisfacción laboral de los trabajadores (Spector, 2016) y, en general, la calidad de vida de las personas (Tavakoli-Fard, et al., 2016), potenciando por una parte lo positivo tal como lo son la autonomía y el reconocimiento profesional y, por otra, evitando las incidencias negativas que derivan de la autoridad extrema que impacta sobre los programas de promoción, exacerba tensiones internas y ralentiza las tareas rutinarias, entre otros efectos adversos (Ardito, et al., 2014). Como corolario al último punto analizado, la sobrecarga laboral que se manifiesta en la extensión de las jornadas de trabajo, impacta negativamente los niveles de insatisfacción laboral dentro de las organizaciones (Ardito, et al., 2014), efecto que se manifiesta, además, sectorialmente en los servicios de salud pública (Jiménez et. al., 2010). En particular, se observa en las áreas de enfermería acentuando las carencias de tiempo y afectando la vida personal de los trabajadores, todo lo cual repercute, tarde o temprano y directamente, sobre el rendimiento laboral de los trabajadores lo cual genera además, un desequilibrio en los determinantes de la salud que abre espacios a la investigación (Greco, 2014).

En un estudio realizado por Pérez y Zurita (2014), en el que se midió CVL y SL en los servicios de salud pública en Chile, se pudo determinar que la relación entre éstas es alta y que elementos como la realización personal, el agotamiento emocional y la despersonalización afectan la CVL. Los estudios confirman el hecho que la SL (Granados, 2011) es clave para incrementar el rendimiento laboral (Daubermann y Tonete, 2012) y que además incide multifactorialmente sobre la CVL, en tanto este último se define como un macro concepto de gestión integral que engloba componentes objetivos y subjetivos del ambiente que afectan directamente las percepciones de los trabajadores (Granados, 2011). Precisamente porque la salud es esencial en la calidad de vida del ser humano, es relevante medir los impactos psicosociales en el trabajo (Stavroula y Aditya, 2013) en consecuencia, el estado de salud de las personas es fundamental y requiere de prestaciones institucionalizadas (González, 2006) de salud física y emocional (Daubermann y Tonete, 2012) a cargo de un sistema público de salud que se ocupa de los impactos adversos sobre las personas (Vidal, et al., 2017) funcional y debidamente focalizado, que tomen a su cargo no solo las necesidades de los usuarios que atiende (Jiménez, et al., 2010), sino que además se ocupe de sus propios personales que fungen como prestadores de dichos servicios de salud (Minsal, 2017).

En el caso particular de Chile, los Centros de Salud Familiar, CESFAM, son los encargados de proveer los cuidados básicos de salud a todos los ciudadanos (Minsal, 2012) y tienen la función de anticiparse a las enfermedades a través de un modelo de atención preventivo que atenúe impactos sobre los personales (Vidal, et al., 2017), cuyo objetivo principal es el de entregar una atención de calidad y, que para lograrlo, cuentan con políticas de mejoramiento que no solo deberán estar orientadas a los pacientes, sino que también deberán orientarse a generar un clima interno satisfactorio (Daubermann y Tonete, 2012), principalmente en relación con los aspectos de la calidad de vida laboral de los trabajadores (Minsal, 2017)

Desde una perspectiva de corto plazo, se necesita gestionar los factores que definen la satisfacción laboral (Granados, 2011) y para ello es preciso potenciar los factores que propician los sentimiento de satisfacción cuyo efecto inmediato se manifiesta en rendimiento laboral (Daubermann y Tonete, 2012). En tanto que, desde una perspectiva de largo plazo, se requiere de la gestión de los componentes que dan forma a una calidad de vida multidimensional, que depende del entorno laboral, de la consecución de objetivos personales y profesionales y de los elementos estructurales que conforman las condiciones de trabajo (Pérez y Zurita, 2014), sea a nivel intangible como la CVL psicológica y, tangible que proviene de la percepción de los medios y recursos disponibles que reportan satisfacción, bienestar y salud (Segurado y Agulló, 2002). Finalmente, sobre la base de los conceptos analizados en torno a la SL y CVL, el presente trabajo busca determinar la incidencia de las dimensiones independientes sobre las dependientes de la CVL, a partir de las percepciones de los trabajadores de la salud primaria que se desempeñan en nueve centros de salud familiar de la Región del Maule, Chile. 


\section{METODOLOGÍA}

La presente investigación es de carácter no experimental, exploratorio, descriptivo y correlacional (Hernández et al., 2006). El universo está integrado por los funcionarios que se desempeñan en 9 centros de salud familiar, CESFAM de la ciudad de Talca, Chile. La muestra determinada a priori previó una varianza máxima de $50 \%$ considerando una distribución normal de la población. Se contempló un intervalo de confianza $\sigma_{P}$ de $95 \%$ (1 - b probabilidad de error) equivalente a una longitud aproximada $Z=1,96$ y se consideró un error de estimación de $5 \%$ como el máximo dispuesto a admitir para el estudio, alcanzándose una muestra representativa estimada de 384 unidades. El plan de muestreo incluyó a empleados, directivos, profesionales, técnicos, administrativos y auxiliares.

\section{Procedimiento}

El cuestionario fue aplicado personalmente a funcionarios de 9 CESFAM, ocho pertenecientes a la ciudad de Talca y uno a la Región Metropolitana, de ambos sexos y que cumplen diversas labores dentro de los centros de salud primaria (Eyssautier, 2006); así mismo, para asegurar la aplicación de las normas de ética en las investigaciones, se optó por aplicar el cuestionario en forma anónima bajo el principio de consentimiento informado (Hernández et al., 2006). El análisis de los datos se realizó resguardando la identidad, tratando de manera agrupada y con absoluta reserva la información de los respondientes (Eyssautier, 2006). Además, con el propósito de asegurar calidad en la recogida de datos, evitar el estrés de los entrevistados y dada la envergadura del instrumento, la duración promedio de respuesta fue de aproximadamente 20 a 30 minutos (González, et al., 2010). El levantamiento de los datos y generación de las bases de datos se realizó entre los meses de septiembre y octubre de 2017.

\section{Instrumento de medición}

El instrumento para la recolección de datos fue el cuestionario de calidad de vida en el trabajo, CVTGOHISALO (González, et al., 2010) que consta con 74 ítems (reactivos) y mide la calidad de vida laboral en siete dimensiones (Tabla 1) y utiliza escala Likert de 0 a 4 puntos, donde 0 es nada satisfecho, nunca, nada de acuerdo o nulo compromiso y 4 indica máxima satisfacción, siempre, nada de acuerdo y total compromiso. El procesamiento estadístico se realizó con el software SPSS versión. 23.0 para el análisis descriptivo, FACTOR Analysis para el análisis factorial exploratorio y AMOS de SPSS, para el análisis factorial confirmatorio.

Tabla 1. Dimensiones del instrumento y sus respectivas preguntas

\begin{tabular}{|l|l|c|}
\hline Dimensión & $N^{\circ}$ de pregunta asociada & $\alpha$ Cronbach dimensión \\
\hline Soporte Institucional & $6-19-26-27-28-44-45-46-47-48-49-50-51-52$ & 0,941 \\
\hline Seguridad en el Trabajo & $5-7-8-9-10-11-12-13-29-30-31-33-61-63-72$ & 0,850 \\
\hline Integración al puesto de trabajo & $18-32-36-37-39-40-41-42-43-65$ & 0,869 \\
\hline Satisfacción por el trabajo & $1-2-3-4-14-15-16-17-20-21-22$ & 0,825 \\
\hline Bienestar logrado a través del trabajo & $23-24-34-35-38-59-60-62-66-71-73$ & 0,805 \\
\hline Desarrollo personal del trabajador & $53-54-64-67-68-69-70-74$ & 0,800 \\
\hline Administración del tiempo libre & $25-55-56-57-58$ & 0,664 \\
\hline Alfa de Cronbach del instrumento & & 0,960 \\
\hline
\end{tabular}

\section{Análisis factorial exploratorio AFE}

Con la finalidad de determinar el número adecuado de factores y definir cuáles van a ser los reactivos que determinan cada dimensión latente, se aplicó la metodología de análisis factorial exploratorio AFE (Freiberg, et al. 2013; Lloret et al. 2014) y se estandarizaron los datos (Freiberg, et al., 2013), para comparar de manera pertinente las variables insertas en la matriz de correlaciones. Para ello se utilizó el método de análisis para variables policóricas (Likert, 0 - 4) y análisis paralelo (PA) con implementación óptima (Timmerman y LorenzoSeva, 2011) el cual permite conseguir auto valores para cada uno de los factores mayores de los que se lograrían al azar. Además se aplicó el método de extracción de mínimos cuadrados no ponderados, ULS, el cual minimiza la suma de los cuadrados de las diferencias entre las matrices de correlaciones observada y reproducida, sin considerar los componentes de la diagonal. Se aplicó rotación oblicua, direct oblimin, haciendo posible asumir la existencia de algún grado de relación entre los factores determinados (Bándalos 
y Finney, 2010), lo que permite a su vez superar las exigencias de la rotación ortogonal que exige independencia de factores, como es habitual que se verifique en estudios de esta naturaleza (Toledo, et. al., 2010). Por otro lado, siguiendo las especificaciones del análisis factorial para la selección de reactivos, se optó por comunalidades altas $(\geq 0,3)$ y cargas factoriales superiores $(\geq 0,5)$ con la finalidad de descartar reactivos con baja correlación (Freiberg, et al., 2013). Seguidamente, se analizó la confiabilidad de los instrumentos, a través del alfa de Cronbach $(\alpha \geq 0,8)$ (George y Mallery, 2003), buscando asegurar la consistencia interna, estabilidad y precisión de los constructos del modelo en estudio. También se determinó la medida de adecuación KMO $(\geq 0,8)$ de Kaiser (Freiberg, et al., 2013), y la prueba de esfericidad de Barlett (Lloret et al., 2014) y se analizaron los gráficos de sedimentación y la determinación cuantitativa de varianza explicada (Borsboom et. al., 2003), con el fin de obtener una solución más simple, interpretable y próxima de la realidad.

\section{Análisis Factorial Confirmatorio AFC y bondad de ajuste}

Ejecutado los pasos para el AFE sugeridos por Freiberg, et al. (2013), se decidió utilizar los métodos de estimación de máxima verosimilitud (ML) y mínimos cuadrados no ponderados (ULS) para ejecutar el análisis factorial confirmatorio (AFC) y ratificar las variables latentes del modelo (Freiberg, et al., 2013). Los análisis de bondad de ajuste se realizaron considerando el índices absoluto estadístico-radio de verosimilitud de chicuadrado (CMIN/DF $\leq 3)$ (Freiberg, et al., 2013), el índice GFI $(\geq 0,9)$, el error de aproximación cuadrático medio RMSEA $(\leq 0,05)$ (Kline, 2005), el índice de error cuadrático medio RMR $(\approx 0)$ y las medidas de ajuste incremental índice ajustado de bondad AGFI $(\geq 0,8)$ (Freiberg, et al., 2013), índice no normado de ajuste 0 índice de Tucker Lewis TLI $(\geq 0,9)$ el índice comparativo de ajuste CFI $(\geq 0,95)$ y el índice normado de ajuste NFI $(\geq 0$,9) (Byrne, 2001; Freiberg, et al., 2013).

\section{RESULTADOS}

En primer lugar, dado que la muestra final post hoc obtenida fue de $408 \geq 384$, determinada a priori, se logró un parámetro de no centralidad de probabilidades 3,229, un valor t critico de 1,648 que define una región crítica o de rechazo y una potencia de 0,943 que define la probabilidad de la prueba estadística para rechazar una hipótesis nula falsa. Además se redujo el error estimado ex ante de $5 \%$ a $4,73 \%$ post hoc. Adicionalmente, se realizó la depuración a la muestra inicial a fin de determinar casos atípicos o datos perdidos, lo que se efectuó mediante el análisis de los casos ejecutando el análisis de caja de SPSS, lo que permitió identificar 105 casos anómalos y confirmar 303 casos depurados.

\section{Descripción de la muestra}

En general, se aprecia que la mayoría de la población encuestada se concentra en el CESFAM Carlos Trupp, con un 15,8\%, seguido por Astaburuaga con un 15,5\% y por Alhué y Magisterio, ambos con un $12,5 \%$ respectivamente. Por otro lado, el $76,2 \%$ de la muestra son Mujeres, mientras que el $23,8 \%$ restante son hombres y donde la mayor población se ubica en el rango de edad entre 29 - 38 años (37,3\%), seguido de las edades entre los 18 y 28 años (20,9\%). Además, el 55,1\% se manifiesta en el ítem de solteros, mientras que el $36,6 \%$ se registra como casados. La antigüedad en sus funciones señala que hasta 5 años se ubicó el $50,5 \%$ y entre 6 a 10 años, se identificó un 20,5\%. La menor concentración de 5,9\% se encuentra entre los 59 y 68 años, con una antigüedad ente los 16 y 20 años de servicio $(4,3 \%)$. Finalmente, la mayoría es profesional $(47,2 \%)$, seguido de Técnicos $(33,7 \%)$ y con cargos administrativos (12,5\%), con turnos fijos $(91,4 \%)$.

\section{Análisis de fiabilidad y validez}

Al verificar la confiabilidad global del instrumento mediante Alfa de Cronbach para la totalidad de los reactivos del instrumento, se obtuvo un coeficiente $\alpha=0,96$ categorizado de muy alto (George y Mallery, 2003). Además, la prueba de esfericidad de Bartlett alcanzó un coeficiente de significancia con valor $p=0,000010$, significativo $(15932,4(\mathrm{df}=2701)$. La medida de adecuación Kaiser-Meyer-Olkin, KMO resultó de 0,93167 calificado de muy bueno $(\geq 0,70)$ el cual sugiere una interrelación satisfactoria para el análisis factorial de los reactivos (Freiberg, et al., 2013).

\section{Análisis Factorial Exploratorio AFE}

Al realizar AFE, se observa en la Tabla 2, la reducción de las dimensiones originalmente planteadas en el instrumento evaluado, quedando solo aquellas variables con cargas factoriales $\geq 0,5$ y comunalidades $\geq 0,3$. El Factor 1 (FCV1), se denomina recreación y familia e incluye los componentes de relación entre el trabajo y el tiempo libre, destacando el poder cumplir con las actividades fuera del trabajo y con la familia. Por su parte, el Factor 2 (FCV2), identificado con el apoyo y reconocimiento institucional, tiene relación con el interés que presenta el jefe directo respecto del subordinado y contiene componentes de reconocimiento del logro, además del apoyo que brinda la institución a sus trabajadores. 
Tabla 2. Factores que influyen en la calidad de vida laboral

\begin{tabular}{|c|c|c|c|c|c|c|c|c|c|}
\hline \multicolumn{4}{|c|}{ Factor 1: Recreación y familia } & \multicolumn{3}{|c|}{$\begin{array}{c}\text { Factor 2: Apoyo y reconocimiento } \\
\text { institucional }\end{array}$} & \multicolumn{3}{|c|}{ Factor 3: Seguridad en el trabajo } \\
\hline Ítem & $\begin{array}{l}\text { Carga } \\
\text { factorial }\end{array}$ & \multicolumn{2}{|c|}{ Comunalidad } & Ítem & $\begin{array}{c}\text { Carga } \\
\text { factorial }\end{array}$ & Comunalidad & Ítem & $\begin{array}{l}\text { Carga } \\
\text { factorial }\end{array}$ & Comunalidad \\
\hline V.62 & 0,558 & \multicolumn{2}{|c|}{0,433} & V.51 & 0,874 & 0,671 & V.16 & 0,555 & 0,344 \\
\hline V.63 & 0,746 & \multicolumn{2}{|c|}{0,601} & V.52 & 0,860 & 0,690 & V.17 & 0,504 & 0,368 \\
\hline V.64 & 0,908 & \multicolumn{2}{|c|}{0,807} & V.53 & \multirow{2}{*}{$\frac{0,803}{0,807}$} & 0,724 & V.19 & 0,686 & 0,593 \\
\hline \multirow[t]{6}{*}{ V.65 } & & \multicolumn{2}{|c|}{0,617} & V.54 & & 0,631 & \multirow[t]{2}{*}{ V.20 } & 0,648 & 0,538 \\
\hline & 0,789 & & & V.55 & 0,819 & 0,681 & & & \\
\hline & & & & V.56 & 0,643 & 0,484 & & & \\
\hline & & & & V.57 & 0,553 & 0,504 & & & \\
\hline & & & & V.58 & 0,545 & 0,522 & & & \\
\hline & & & & V.59 & 0,555 & 0,512 & & & \\
\hline \multicolumn{2}{|c|}{ Varianza explicada } & \multicolumn{2}{|c|}{0,29103} & & & 0,05477 & & & 0,03877 \\
\hline \multicolumn{3}{|c|}{$\%$ varianza de datos reales } & $30,3^{*}$ & & & $5,7^{*}$ & & & $4,0^{*}$ \\
\hline \multicolumn{2}{|c|}{ Alfa de Cronbach } & & 884 & & & 0,936 & & & 0,817 \\
\hline \multicolumn{5}{|c|}{ Factor 4: Bienestar laboral } & & \multicolumn{4}{|c|}{ Factor 5: Desarrollo e integración } \\
\hline \multicolumn{2}{|c|}{ Ítem } & $\begin{array}{l}\text { Carga } \\
\text { Factorial }\end{array}$ & Comu & halidad & & Ítem & & arga Factorial & Comunalidad \\
\hline V.14 & 0,6 & & & 79 & & V.18 & & 0,542 & 0,401 \\
\hline & & & & & & V.37 & & 0,589 & 0,474 \\
\hline V.15 & 0,5 & & & 19 & & $\mathrm{~V}, 72$ & & 0,51 & 0,399 \\
\hline V.36 & 0,6 & & 0,5 & 11 & & V.73 & & 0,621 & 0,437 \\
\hline V.67 & 0,5 & & 0, & 93 & & V.74 & & 0,516 & 0,449 \\
\hline Varianza $\epsilon$ & plicada & & 0,0 & 973 & & & & & 0,02789 \\
\hline$\%$ varianz & de datos real & & & $0^{\star}$ & & & & & $2,9^{*}$ \\
\hline Alfa de $\mathrm{Cr}$ & hbach & & 0,8 & 02 & & & & & 0,753 \\
\hline
\end{tabular}

A su vez, el Factor 3 (FCV3), conserva el nombre original de la dimensión seguridad en el trabajo y tiene relación con los sistemas de seguridad social, planes de retiro, oportunidades de capacitación y la conformidad de los trabajadores respecto de la cantidad de insumos necesarios para cumplir con las funciones que le competen a la institución. En tanto que el factor 4 (FCV4), denominado bienestar laboral, vincula el grado de satisfacción del trabajador con las compensaciones y que se expresan en el sueldo que cada uno perciben en relación con sus pares. Por último, el Factor 5 (FCV5), denominado desarrollo e integración, establece una relación entre la integridad del trabajador, la percepción de un trabajo acorde a la preparación académica exigida, la contribución a la imagen institucional que conlleva y el grado de compromiso de ésta para lograr los objetivos organizacionales. Finalmente, los cinco factores que conforman el constructo de la calidad de vida laboral validado, CVLV, representan un $42,9 \%$ de la varianza total explicada.

\section{Análisis Factorial Confirmatorio AFC}

Para el análisis de covarianzas se aplicaron los métodos máxima verosimilitud, ML y mínimos cuadrados no ponderados, ULS. La especificación del modelo se muestra en la Figura 1 en la que se puede visualizar las relaciones de las dimensiones del constructo con sus respectivos indicadores.

En términos generales las relaciones entre los constructos son significativas y directas. Se observa que los factores con mayor influencia corresponden a apoyo y reconocimiento Institucional (FCV2) y seguridad en el trabajo (FCV3), los que se relacionan directamente con desarrollo e integración (FCV5) (FCV2 <-> FCV5 = 0,489 y FCV3 <-> FCV5 $=0,417$ ). Por otro lado, los factores que presentan una menor influencia entre sí, son los relacionados con el constructo apoyo y reconocimiento institucional (FCV2), siendo estos recreación y familia (FCV1 <-> FCV2 = 0,119) y bienestar laboral (FCV4 <-> FCV2 $=0,298)$. 


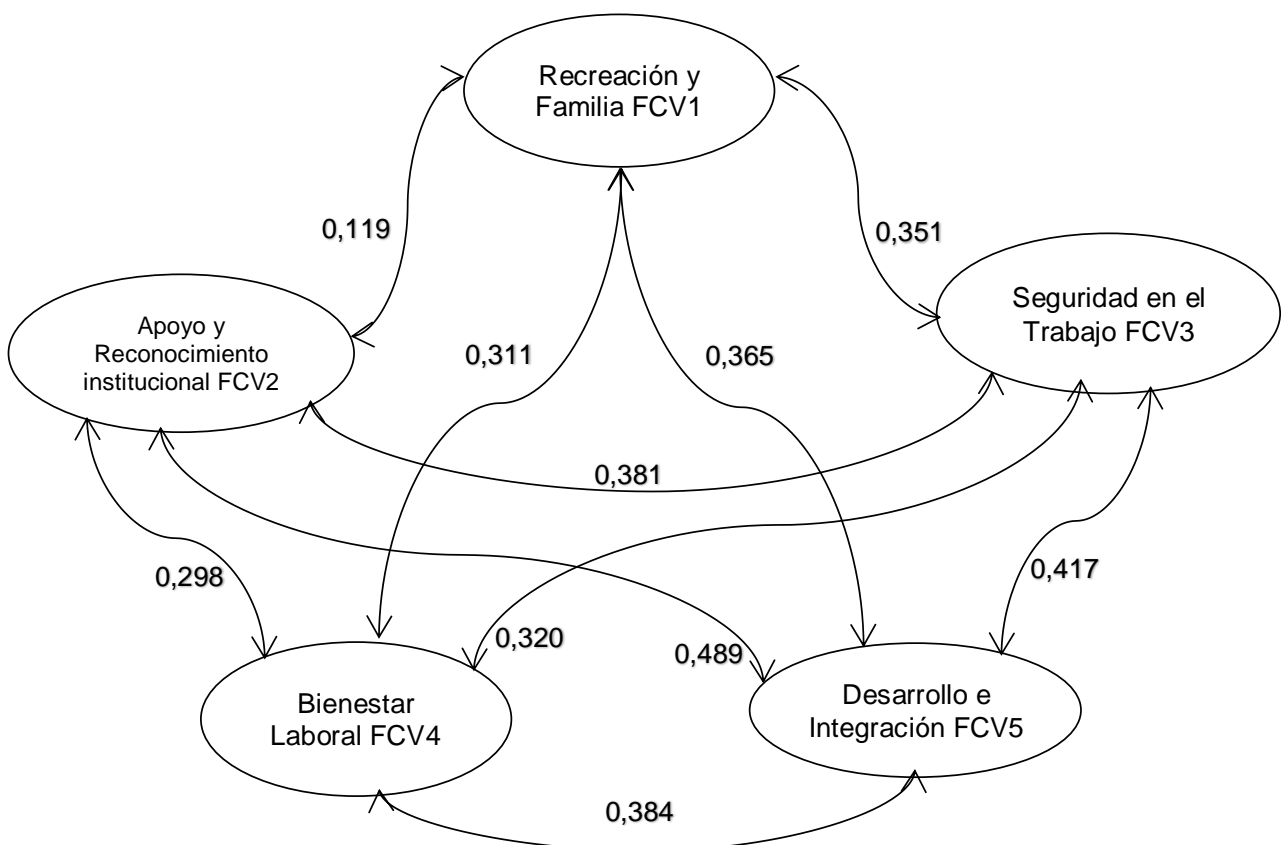

Fig. 1: Modelo Global de covarianzas ajustado y estandarizado por método ML

Por otra parte, el detalle de los resultados del análisis confirmatorio se presentan en la tabla 3 , dónde se muestra el modelo relacional de covarianzas de las variables latentes de calidad de vida laboral. FCV1: recreación y familia, FCV2: apoyo y reconocimiento institucional, FCV3: seguridad en el trabajo, cuyos indicadores son endógenos ya que son determinados por sus respectivas variables latentes y, en el caso de éstas últimas, FCV4: bienestar laboral y FCV5: desarrollo e integración, son definidas como exógenas puesto que no son determinados por ninguna otra variable.

Tabla 3: Parámetros de las variables Modelo Covarianzas Ajustado

\begin{tabular}{|c|c|c|c|c|c|c|}
\hline & \multicolumn{3}{|c|}{$\mathrm{ML}$} & \multicolumn{3}{|c|}{ ULS } \\
\hline & $\begin{array}{c}\text { Peso de la } \\
\text { regresión } \\
\beta_{1}\end{array}$ & $\begin{array}{c}\text { Varianza } \\
\quad \beta_{2}\end{array}$ & $\begin{array}{c}\text { Valor } \\
\text { Estándar }\end{array}$ & $\begin{array}{c}\text { Peso de la } \\
\text { regresión } \\
\beta_{1}\end{array}$ & $\begin{array}{c}\text { Varianza } \\
\beta_{2}\end{array}$ & $\begin{array}{c}\text { Valor } \\
\text { Estándar }\end{array}$ \\
\hline V16 $\leftarrow$ FCV3 & $1,000^{\star * \star}$ & $0,832^{\star * *}$ & 0,515 & $1,000^{\star * *}$ & $0,519^{* * *}$ & 0,827 \\
\hline $\mathrm{V} 17 \leftarrow \mathrm{FCV} 3$ & $1,171^{* * *}$ & $1,044^{* * *}$ & 0,531 & $1,309^{* * *}$ & $0,599^{* * *}$ & 0,934 \\
\hline $\mathrm{V} 19 \leftarrow \mathrm{FCV} 3$ & $1,565^{\star * \star}$ & $0,236^{\star * *}$ & 0,870 & $1,474^{* * *}$ & $0,826^{* * *}$ & 0,309 \\
\hline V20 $\leftarrow$ FCV3 & $1,697^{\star * *}$ & $0,252^{\star \star *}$ & 0,880 & $1,623^{\star * *}$ & $0,848^{* * *}$ & 0,313 \\
\hline V52 $\leftarrow$ FCV2 & $1,000^{* * *}$ & $0,203^{\star * *}$ & 0,847 & $1,000^{* * *}$ & $0,793^{* * *}$ & 0,271 \\
\hline V51 $\leftarrow$ FCV2 & $0,947^{* * *}$ & $0,230^{\star * *}$ & 0,818 & $0,957^{\star \star \star}$ & $0,779^{* * *}$ & 0,271 \\
\hline V53 $\leftarrow$ FCV2 & $1,061^{* * *}$ & $0,118^{* * *}$ & 0,912 & $1,023^{\star * *}$ & $0,826^{* * *}$ & 0,224 \\
\hline V54 $\leftarrow$ FCV2 & $1,063^{* * *}$ & $0,230^{\star * *}$ & 0,847 & $1,026^{* * *}$ & $0,768^{* * *}$ & 0,334 \\
\hline V55 $\leftarrow$ FCV2 & $0,931^{* * *}$ & $0,155^{\star \star \star}$ & 0,863 & $0,948^{\star * *}$ & $0,826^{\star * * *}$ & 0,192 \\
\hline V56 $\leftarrow$ FCV2 & $0,858^{* * *}$ & $0,251^{* * *}$ & 0,776 & $0,831^{* * *}$ & $0,709^{* * *}$ & 0,314 \\
\hline V57 $\leftarrow$ FCV2 & $0,824^{* * *}$ & $0,458^{\star * *}$ & 0,659 & $0,984^{* * *}$ & $0,740^{* * *}$ & 0,367 \\
\hline V58 $\leftarrow$ FCV2 & $0,854^{* * *}$ & $0,591^{* * *}$ & 0,625 & $1,071^{* * *}$ & $0,732^{* * *}$ & 0,457 \\
\hline V59 $\leftarrow$ FCV2 & $0,932^{\star * \star}$ & $0,527^{\star \star \star}$ & 0,679 & $1,141^{\star * *}$ & $0,782^{\star \star \star}$ & 0,379 \\
\hline V76 $\leftarrow$ FCV4 & $1,000^{\star * *}$ & $0,840^{* * *}$ & 0,599 & $1,000^{\star \star \star}$ & $0,680^{\star \star *}$ & 0,704 \\
\hline V36 $\leftarrow$ FCV4 & $1,267^{* \star *}$ & $0,316^{\star \star \star}$ & 0,839 & $1,009^{\star * *}$ & $0,760^{\star \star \star}$ & 0,451 \\
\hline $\mathrm{V} 15 \leftarrow$ FCV4 & $0,911^{* * *}$ & $0,553^{\star * *}$ & 0,643 & $0,815^{\star \star *}$ & $0,653^{\star * \star}$ & 0,541 \\
\hline $\mathrm{V} 14 \leftarrow \mathrm{FCV} 4$ & $0,932^{\star * *}$ & $0,371^{* * *}$ & 0,744 & $0,789^{\star * *}$ & $0,673^{\star \star \star}$ & 0,455 \\
\hline V65 $\leftarrow$ FCV1 & $1,000^{\star \star \star}$ & $0,250^{\star \star \star}$ & 0,837 & $1,000^{\star * *}$ & $0,763^{\star \star \star}$ & 0,348 \\
\hline V64 $\leftarrow$ FCV1 & $1,114^{* * *}$ & $0,136^{\star * *}$ & 0,918 & $1,205^{\star * *}$ & $0,905^{\star \star *}$ & 0,156 \\
\hline V63 $\leftarrow$ FCV1 & $0,846^{\star \star \star}$ & $0,247^{\star \star \star}$ & 0,792 & $0,911^{\star * *}$ & $0,778^{\star \star \star}$ & 0,262 \\
\hline $\mathrm{V} 62 \leftarrow$ FCV1 & $0,730^{\star \star \star}$ & $0,396^{\star * *}$ & 0,663 & $0,960^{\star * *}$ & $0,795^{\star * *}$ & 0,260 \\
\hline V67 $\leftarrow$ FCV5 & $1,000^{* * *}$ & $0,159^{* * *}$ & 0,381 & $1,000^{* * *}$ & $0,287^{* * *}$ & 0,171 \\
\hline V72 $\leftarrow$ FCV5 & $3,251^{* \star *}$ & $0,327^{\star \star \star}$ & 0,683 & $4,630^{\star * *}$ & $0,733^{\star \star \star}$ & 0,283 \\
\hline V73 $\leftarrow$ FCV5 & $2,271^{* * *}$ & $0,201^{* * *}$ & 0,639 & $2,700^{* * *}$ & $0,573^{* * *}$ & 0,228 \\
\hline V74 $\leftarrow$ FCV5 & $3,699^{* * *}$ & $0,263^{* * *}$ & 0,764 & $4,936^{\star * *}$ & $0,769^{* * *}$ & 0,258 \\
\hline V81 $\leftarrow$ FCV5 & $2,086^{* * *}$ & $0,169^{\star * *}$ & 0,640 & $2,720^{* * *}$ & $0,630^{* * *}$ & 0,173 \\
\hline
\end{tabular}


Tabla 3: (continuación)

\begin{tabular}{|c|c|c|c|c|}
\hline \multicolumn{5}{|c|}{ Parámetros modelo Covarianzas Ajustado } \\
\hline & Estimador & Estándar & Estimador & Estándar \\
\hline FCV3 <-> FCV2 & $0,150^{\star \star *}$ & 0,381 & 0,169 & 0,454 \\
\hline FCV3 <-> FCV4 & $0,120^{\star \star *}$ & 0,320 & 0,173 & 0,403 \\
\hline FCV3 <-> FCV1 & $0,147^{\star * *}$ & 0,351 & 0,150 & 0,391 \\
\hline FCV3 <-> FCV5 & $0,038^{\star * *}$ & 0,417 & 0,029 & 0,418 \\
\hline FCV2 <-> FCV4 & $0,147^{\star * *}$ & 0,298 & 0,196 & 0,190 \\
\hline FCV2 <-> FCV1 & $0,065^{\star}$ & 0,119 & 0,090 & 0,527 \\
\hline FCV2 <-> FCV5 & $0,058^{\star \star *}$ & 0,489 & 0,044 & 0,354 \\
\hline FCV4 <-> FCV1 & $0,163^{\star \star *}$ & 0,311 & 0,192 & 0,459 \\
\hline FCV4 <-> FCV5 & $0,043^{\star \star *}$ & 0,384 & 0,044 & 0,385 \\
\hline FCV1 <-> FCV5 & $0,046^{\star \star *}$ & 0,365 & 0,033 & \\
\hline
\end{tabular}

Los resultados obtenidos pueden explicarse por el contenido conformado por los componentes de cada constructo o dimensión (Lloret et al., 2014), otorgándole a cada factor su respectiva naturaleza y sentido y, por lo mismo, le proporcionan capacidad de relación (Freiberg, et al., 2013) con las demás dimensiones del modelo, dando forma a un nuevo cuestionario de calidad de vida laboral validado, CVLV. En el caso particular de los factores con menor incidencia, la primera relación (FCV1 <-> FCV2) puede explicarse, en cierta medida por el apoyo y reconocimiento institucional que busca expresar cómo las instituciones de salud pública enfrentan la calidad del trabajo o servicio que es entregado por el funcionario, manifestándose en reconocimiento a las aptitudes, cualificaciones, desempeño y competencias que posee cada trabajador en su labor. Por otra parte, el constructo recreación y familia, se relaciona con la posición que tiene, tanto el funcionario como la institución sobre el ámbito familiar, la disposición de tiempos, empatía y diversos temas relacionados con este factor.

\section{Medidas de ajuste}

Se observa en la tabla 4 que los resultados en relación con la bondad de ajuste absoluto, muestra que el estadístico CMIN/DF para el método ML y ULS sin ajuste, es 2,798 y 0,841 respectivamente, indicando que previo al ajuste el modelo está correctamente especificado (Freiberg, et al., 2013), el cual una vez ajustado, toma valores de 1,658 para el modelo ML y 0,682 para el ULS confirmando la adecuación del modelo con la realidad que busca representar. Por otro lado, los índices de error que corroboran la adecuación del modelo muestran un índice RMSEA que alcanza para el método análisis $M L$ una puntuación de $0,047(\leq 0,5)$ y un índice CFI de 0,96 ( $\geq 0,85)$, indicando que existe un buen ajuste de los constructos (Freiberg, et al., 2013).

Tabla 4: Bondad de Ajuste

\begin{tabular}{|l|c|c|c|c|}
\hline & ML sin ajuste & ML con ajuste & ULS sin ajuste & ULS con ajuste \\
\hline CMIN/DF & 2,798 & 1,658 & 0,841 & 0,682 \\
\hline CMIN & 808,609 & 456,003 & 242,933 & 1887,582 \\
\hline DF & 289 & 275 & 289 & 275 \\
\hline RMR & 0,057 & 0,053 & 0,048 & 0,042 \\
\hline GFI & 0,825 & 0,900 & 0,975 & 0,980 \\
\hline AGFI & 0,787 & 0,871 & 0,969 & 0,975 \\
\hline NFI & 0,835 & 0,907 & - & - \\
\hline TLI & 0,872 & 0,953 & - & - \\
\hline CFI & 0,887 & 0,960 & - & - \\
\hline RMSEA & 0,077 & 0,047 & - & - \\
\hline
\end{tabular}

Por último, en términos generales, la bondad de ajuste en ambos métodos es consistente y define una correcta especificación del modelo planteado, siendo representativo del modelo real. Los índices GFI $(0,9=0,9)$, AGFI $(0,871 \geq 0,8)$ son considerados satisfactorios (Freiberg, et al., 2013), y adicionalmente, los índices NFI $(0,907$ $\geq 0,9)$ y TLI $(0,953 \geq 0,9)$ son considerados excelentes (Byrne, 2001; Freiberg, et al., 2013). Lo cual demuestra que el modelo propuesto logra una aproximación pertinente con la realidad que busca representar. 


\section{DISCUSIÓN}

Los resultados del análisis realizado permite afirmar que, efectivamente, los recursos intangibles son de vital importancia para las organizaciones y por lo mismo validan la necesidad de medir la CVL de los trabajadores (Daubermann y Tonete, 2012), considerando las perspectivas objetiva y subjetiva que la definen (Granados, 2011).

Debido a que el FCV2: apoyo y reconocimiento institucional muestra la mayor correlación positiva con el FCV5: desarrollo e integración, es que se hace crucial incorporar este hallazgo entre las principales decisiones destinadas a la gestión de personas (Granados, 2011). Así también se ratifica la idea que tiene el trabajador de lo que recibirá como recompensa al finalizar su trabajo (Daubermann y Tonete, 2012). Por una parte, porque entre las primeras acciones de una persona, está el de comenzar con una evaluación subjetiva acerca de la probabilidad de que la organización valore su desempeño y le entregue las recompensas que estima merecer (Granados, 2011), ya sean del tipo primario, como aumentos de sueldo o secundario, como estatus, oportunidades y reconocimiento (Granados, 2011).

En síntesis, la relación entre las compensaciones como expresión de sentido, potencian tanto a la organización como al trabajador individual, en la medida en que se incremente la calidad de vida laboral (Minsal, 2017). La gestión eficaz de este componente promueve motivaciones individuales intrínsecas, que son las encargadas de que el desempeño sea sobresaliente y sostenido en el tiempo (Granados, 2011). Los hallazgos del presente trabajo confirman el estudio realizado por Pérez y Zurita, (2014), en el que se relacionan CVL y SL en los servicios de salud pública en Chile, determinando que la relación entre éstas es alta y que elementos como realización personal, agotamiento emocional y despersonalización, se ven afectados cuando son relacionados con la CVL (Kok, et al., 2014) y el hecho de interpretar que elementos externos como los recursos destinados para la atención y los insumos (Granados, 2011) afectan claramente el nivel y calidad de respuesta de las atenciones públicas y principalmente la CVL de los funcionarios, puede colaborar en evitar sobrecargas y estrés laboral (Granados, 2011; Minsal, 2017).

Lo anterior demuestra, a grandes rasgos, que elementos externos al clima laboral como los recursos y los insumos (Segurado y Agulló, 2002), son capaces de afectar las relaciones dentro de cualquier organización, provocando graves cambios en las actitudes de los funcionarios y sobretodo en su salud física y mental (Daubermann y Tonete, 2012). Por otra parte, la CLV descrita en la presente investigación ratifica, en términos de los factores determinados, los estudios de Patlán (2016), quien propone 16 constructos con un factor global, utilizando las ideas planteadas por Segurado y Agulló (2002). En esta misma línea de análisis, el presente trabajo confirma los hallazgos de Hernández et. al (2017), quienes aplicaron los cuestionarios QLABORS 56, CVT-GOHISALO y CVL-SA-8000, determinando que bienestar individual, condiciones y medio ambiente de trabajo, organización y bienestar logrado a través del trabajo, constituyen dimensiones claves de la CVL en hospitales y centros de salud.

Finalmente es importante mencionar que el presente estudio exhibe una reducción de los reactivos relevantes del cuestionario original CVL-GOHISALO (Hernández et. al., 2017), luego de ser aplicados en los centros prestadores de salud primaria CESFAM en la Zona Central de Chile, dando como resultado 5 factores que contienen a las 26 reactivos o ítems persistentes que alcanzan índices de confiabilidad adecuada para su aplicación al interior de los centros de salud chilenos (González et al., 2010) dando forma a un nuevo cuestionario de calidad de vida laboral validado, CVLV. Estos hallazgos ponen a disposición de los directivos de los centros de salud primaria, un instrumento que permite la recogida de información crucial y compatible con los requerimientos de gestión institucional (Minsal, 2017), para la toma de decisiones en torno a la calidad de vida laboral en relación con los elementos centrales de las acciones sanitarias en manos de los personales que los desempeñan (Costa, et al., 2013) y respecto de factores individuales y contextuales que finalmente inciden en el desempeño de los trabajadores de la salud (Kawakatsu, et al., 2015).

\section{CONCLUSIONES}

Se identificaron las variables que influyen en la percepción que tienen los funcionarios sobre calidad de vida laboral al interior de los Centros de Salud Familiar de la zona central de Chile, determinando que existe relación entre las variables y que los respectivos constructos covarian alcanzando índices positivos y significativos.

Se determinó que los factores recreación y familia, apoyo y reconocimiento institucional, seguridad, bienestar laboral y desarrollo e integración, inciden sobre la percepción de calidad de vida laboral de los funcionarios de la salud primaria. Las relaciones de mayor incidencia se presentaron entre las dimensiones seguridad y trabajo y apoyo y reconocimiento institucional respecto de desarrollo e integración. Por el contrario, las relaciones de menor incidencia se presentaron entre los factores recreación y familia y bienestar laboral respecto de apoyo y reconocimiento laboral. 
Finalmente, los índices de bondad de ajuste permiten concluir que el modelo teórico o hipotético estimado de calidad de vida laboral que sostiene el cuestionario de calidad de vida laboral validado, es aceptable, dado que alcanza un buen ajuste al comportamiento de los datos de la realidad que busca representar.

\section{REFERENCIAS}

Ardito C., D'Errico A. y Leombruni R., Exposure to Psychosocial Factors at Work and Mental Well-Being in Europe. La Medicina del Lavoro. 105(2):85-99 (2014).

Bandalos, D. L. y Finney, S. J., Factor Analysis: Exploratory and Confirmatory. En G. R. Hancock y R. O. Mueller (Eds.), Reviewer's guide to quantitative methods. Routledge. New York (2010).

Borsboom, D., Mellenbergh G. J. y Van Heerden, J., The Theoretical Status of Latent Variables. Psychological review, 110(2), 203 (2003).

Costa, S. de M., Araújo, F. F. y otros 4 autores, Community health worker: a core element of health actions. Ciên Saude Colet. 18(7):2147-56 (2013).

Daubermann, D. C. y Tonete, V. L. P., Quality of work life of nurses in primary health care. Acta Paulista de Enfermagem, 25(2), 277-283. (2012).

Eyssautier, M., Metodología de la Investigación. México D.F.: Editorial Thomson (2006)

Freiberg, H. A., Stover, J. B., de la Iglesia, G., y Fernández L. M., Polychoric And Tetrachoric Correlations In Exploratory And Confirmatory Factorial Studies. Prensa Médica Latinoamericana. Ciencias Psicológicas, VII (2) 151 -164 (2013)

George, D. y Mallery, P., SPSS for Windows step by step: A Simple Guide and Reference. 11.0 Update (4..$^{\text {a }}$ ed.). Boston: Allyn \& Bacon (2003)

González, B., Hidalgo, S., Salazar, E., Preciado, S., Elaboración y Validación del Instrumento para Medir Calidad de Vida en el Trabajo CVT-GOHISALO. Ciencia \& Trabajo. Abril - Junio; 12 (30) 332-340 (2010).

Granados, I., Calidad de Vida Laboral: Historia, Dimensiones y Beneficios. Revista IPS, Facultad de Psicología, Universidad Nacional Mayor de San Marcos. 14 (2) 271-276. Lima, Perú. (2011).

Greco, E., V. Workshop. Health Determinants: New Paths for Scientific Research.Istisan Congressi 14/C2. ISBN: 03935620. Istituto Superiore di Sanità, Rome, Italy, Istisan Congressi 14/C2 edition, May 8, (2014).

Hernández, I., Guzmán, M., y otros 4 autores, Validación de una Escala para Medir la Calidad de Vida Laboral en Hospitales Públicos de Tlaxcala. Salud Pública de México, 59 (2) 183-192 (2017).

Hernández, R., Fernández C. y Baptista, P., Metodología de la Investigación (4. ${ }^{a}$ ed.) McGraw-Hill, México D. F. (2006)

Jiménez, A., D. Acevedo, L. Salgado, E. Moyano. Cultura Trabalho-Família e Compromisso Organizacional numa Empresa de Serviços. Psicol Estud. 14(4) 729-38 (2010).

Kawakatsu, Y., Sugishita, T. y otros 4 autores, Individual and Contextual Factors Associated with Community Health Workers' Performance in Nyanza Province, Kenya: a multilevel analysis. BMC Health Serv Res. 15(1):442 (2015).

Kline, R. Principles and Practice of Structural Equation Modeling. New York: Gilford Press (2005).

Kok, M.C., Dieleman, M. y otros 4 autores, Which Intervention Design Factors Influence Performance of Community Health Workers in low- and Middle-Income Countries? A systematic review. Health Policy and Planning Advance. 1-21 (2014).

Lloret, S., Ferreres, A., Hernández A. y Tomás, I., El Análisis Factorial Exploratorio de los Ítems: una Guía Práctica, Revisada y Actualizada. Anales de psicología, 30(3), 1151-1169 (2014).

MINSAL., Orientación para la Implementación del Modelo de Atención Integral de Salud Familiar y Comunitaria. Subsecretaria de Redes Asistenciales, División Atención Primaria, Ministerio de Salud. Chile (2012).

MINSAL., Plan de Calidad de Vida Laboral 2015 - 2018. Servicio de Salud Metropolitano Suroriente, Subdirección de las personas, Departamento de Calidad de Vida Laboral. Chile (2017).

Patlán, J., Construcción y Propiedades Psicométricas de la Escala de Calidad de Vida en el Trabajo. Revista Ciencia y Trabajo. Año 18 N56 94-105 (2016).

Pérez, Z. D. y Zurita, R., Calidad de Vida Laboral en Trabajadores de Salud Pública en Chile. Salud \& Sociedad. 5(2) $172-$ 180 (2014).

Segurado, A. y Agulló, E., Calidad de Vida Laboral: Hacia un Enfoque Integrador desde la Psicología Social. Psicothema, 14(4) 828-836 (2002).

Sirgy, M. J., Reilly N. P. y Wu. J., A Work-Life Identity Model of Well-Being: Towards a Research Agenda Linking Qualityof-Work-Life (QWL) Programs with quality of Life (QQL). Applied Research Quality Life, 3, 181-202 (2008).

Spector, P. E., Industrial and Organizational Psychology Research And Practice.Ipswich, Mass, USA: Wiley; Children's Literature Comprehensive Database. (2016). 
Stavroula L., Aditya J., Health Impact of Psychosocial Hazards at Work: An Overview. Geneva, Switzerland: Publications of the World Health Organization (2013).

Tavakoli-Fard, N., Mortazavi, S. A., Kuhpayehzadeh, J. y Nojomi, M., Quality of Life, Work Ability and Other Important Indicators of Women's Occupational Health. Int J Occup Med Environ Health. 29(1):77-84 (2016).

Timmerman, M. y Lorenzo-Seva, U., Dimensionality Assessment of Ordered Polytomous Items with Parallel Analysis. Psychological Methods, 16. 209-220 (2011).

Toledo V. A., Merino E. J.M., Cruz P. M., Validez Constructiva De La Escala Q-Labors 56 En Enfermeros/As Chilenos/As. Ciencia y Enfermería XVI (3): 41-47 (2010).

Vidal G. C., Palavecino S. I., Moya R. P., Toro H. C. y Hoffmeister A. L., Calidad de Vida del Personal de Salud y su Relación con el Ausentismo. Cienc Trab. Sep-Dic; 19 [60]: 188-193 (2017). 